\title{
Modélisation éthique de l'Internet des Objets
}

\section{Ethic modelisasion for Internet of Thing}

\author{
Samuel Szoniecky ${ }^{1}$, Stéphane Safin ${ }^{2}$ \\ ${ }^{1}$ Laboratoire Paragraphe EA 1573, Université Paris VIII, Saint-Denis France, samuel.szoniecky@univ-paris8.fr \\ 2 Laboratoire Paragraphe EA 1573, Université Paris VIII, Saint-Denis France, stephane.safin@univ-paris8.fr
}

RÉSUMÉ. L'article présente les principes théoriques et graphiques pour une modélisation éthique de l'internet des Objets. Ces principes sont appliqués à la modélisation des points de vue du parlement européen sur la question des règles de droits civiles pour la robotique. Plus particulièrement, nous montrons comment récolter des données brutes pour la modélisation d'existences informationnelles dans un écosystème de connaissances. En conclusion nous nous interrogeons sur la viabilité d'une signalétique pour évaluer l'éthique des objets connectés et sur les travaux qu'ils restent à mener pour y parvenir.

ABSTRACT. The article presents the theoretical and graphical principles for an ethical modeling of the Internet of the Things. These principles are applied to modeling the views of the European Parliament on the issue of civil rights rules for robotics. More specifically, we show how to collect raw data for modeling information existences in a knowledge ecosystem. In conclusion, we question the viability of a diagram to evaluate the ethics of connected objects and the work they still have to do to achieve this.

MOTS-CLÉS. Internet des objets, éthique, ontologie, pouvoir d'agir, diagramme, parlement européen.

KEYWORDS. Internet of things, ethics, ontology, power of action, diagram, European Parliament.

\section{Introduction}

Le développement de l'internet des objets entraine avec lui une redéfinition des frontières du numériques [BOU 2016] qui impactent fortement nos sociétés à la fois d'un point de vue technique, industriel, commercial, social et politique. La prolifération des objets connectés s'observe dans notre quotidien pour des usages de plus en plus variés qui passent essentiellement par nos téléphones mobiles qui se sont transformés en plateforme d'échanges avec nos voitures, nos télévisions ou tout autre objet domestique (réfrigérateur, four, chauffage...). La définition de ces échanges, l'évaluation de leurs finalités et leurs contrôles posent de nombreuses questions [SAL 2017] [NOY 2017] qui ne se cantonnent pas uniquement à des problèmes techniques [YAN 2017] [ALO 2017] mais couvrent plus globalement le champ des usages socio-sémantiques du numérique [BAC 2011].

Pour participer aux réflexions actuelles ${ }^{1}$ et ouvrir un débat démocratique sur les problématiques qu'elles entrainent, nous proposons dans une série d'articles, dont celui-ci est le premier, une méthode de modélisation de l'Internet des Objets qui a pour objectif d'évaluer et de comparer l'éthique de ces technologies. Pour ce faire, nous développons des outils pour la modélisation du pouvoir d'agir de ces objets connectés afin de comprendre leurs impacts sur nos vies quotidiennes. Par exemple, nous réfléchirons à une signalétique simple indiquant le positionnement éthique des objets à la manière des pictogrammes qui informent les consommateurs sur la qualité énergétique des appareils électroménagers. Mais avant d'arriver à l'expression du pouvoir d'agir sous cette forme simplifiée, nous devons nous interroger sur les principes théoriques et graphiques de ces diagrammes ainsi que sur leur mode de conception.

Après avoir posé les cadres théoriques de cette question en montrant que la définition du positionnement éthique passe par la modélisation du pouvoir d'agir dans un écosystème de connaissances suivant quatre dimensions existentielles fondamentales (physiques, acteurs, concepts,

\footnotetext{
${ }^{1}$ Par exemple : https://www.inria.fr/actualite/actualites-inria/transalgo
} 
rapports), nous utiliserons ces principes pour définir une grammaire graphique nous permettant de représenter ces pouvoirs d'agir sous la forme de diagramme. Nous traiterons ensuite la question de la collecte des données brutes nécessaires pour la conception de ces diagrammes. Pour illustrer notre propos nous récolterons les positionnements éthiques du parlement européen en partant des débats concernant les règles de droit civil sur la robotique. Nous terminerons notre propos en nous interrogeant sur la viabilité d'une signalétique éthique construite dynamiquement par des procédures d'intelligence collective.

Face aux enjeux de l'Internet des Objets, les chercheurs et particulièrement les spécialistes de l'ergonomie et des sciences de l'information et de la communication, n'ont-ils pas la responsabilité de fournir au plus grand nombre les moyens de comprendre et de gérer ces écosystèmes de connaissances hyper-complexes?

\section{Principes de modélisation éthique}

Nous avons exposé dans plusieurs articles notre méthode de modélisation en l'appliquant à des problématiques liées aux recherches en humanité numérique [SZO 15a][ SZO 15b] nous ne traiterons pas en détail les principes que nous mettons en œuvre et nous renvoyons les lecteurs désirant en savoir plus à ces articles.

\subsection{Principes théoriques}

Les principes théoriques que nous utilisons s'appuient sur une volonté de modéliser les problématiques d'information - communication à travers l'analogie des écosystèmes afin de tirer partie d'une approche incluant la complexité du vivant dans un domaine traditionnellement analysé avec des modèles figés comme ceux de l'architecture. D'autre part, les écosystèmes offrent des analogies comme celle du jardin qui sont facilement compréhensibles, ce qui permet d'envisager une appropriation «grand public» des modèles et plus encore de leur conception notamment dans une visée pédagogique.

En suivant ce cadre analogique, le travail de modélisation passe par la définition des existences qui peuplent ces écosystèmes et des relations que ces existences entretiennent les unes avec les autres. Pour concevoir ces modélisations existentielles nous utilisons de multiples références ${ }^{2}$ dont voici les principales. Tout d'abord, nous nous inspirons de travaux de Gilles Deleuze sur les propositions de Spinoza pour « une ontologie qui soit le corréla d'une éthique » [DEL 1980]. Pus particulièrement nous reprenons les principes d'une existence composée de trois dimensions (parties extensives, rapports, essences) corrélées à trois genres de connaissance (chocs, rapports, intuitions). Comme autre inspiration importante, nous devons citer Philippe Descola [DES 05] qui avec ces «matrices ontologiques " propose une vision simple de l'existence basée sur la cardinalité des rapports entre physicalités et intériorités. Bruno Latour nous apporte quand a lui une typologie des modes d'existences [LAT 12] qui place l'acteur au cœur d'un réseau où les principes écologiques [LAT 15] permettent de comprendre les processus complexes d'influences. Enfin, citons Pierre Rabardel dont les recherches en analyse de l'activité nous apportent la notion de pouvoir d'agir comme cadre ergonomique [RAB 05].

\subsection{Principes graphiques}

Les principes graphiques que nous utilisons sont très simples, ils se basent sur l'utilisation de quatre figures géographiques (rectangle, hexagone, cercle, trait) représentant chacune une des dimensions existentielles du modèle (physique, acteur, concept, rapport). Contrairement aux représentations construites avec des outils comme GEPHI qui mettent en forme un réseau composé de nœuds et de

\footnotetext{
${ }^{2}$ http://www.samszo.univ-paris8.fr/spip.php?page=biblio
} 
liens, nos principes graphiques de modélisation mettent en jeu quatre réseaux qui se superposent sans dénaturer la dimension existentielle et les caractéristiques propres de chacun de ces réseaux.

\subsubsection{Les dimensions physiques}

Les dimensions physiques correspondent aux «parties extensives» de Spinoza et aux «physicalités » de Descola. Elles sont les dimensions matérielles de l'existence comme par exemple une bibliothèque, une étagère, un livre, sa couverture, ses pages, l'encre du texte... Elles sont soumises aux lois physiques fondamentales et peuvent donc être mesurées. Elles sont représentées par des rectangles avec un titre. Ces rectangles peuvent s'imbriquer pour définir une arborescence de parties.

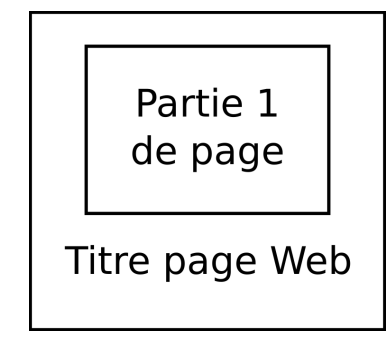

Figure 1. Modélisation des dimensions physiques

\subsubsection{Les acteurs}

Les acteurs sont les entités responsables de la création des rapports dans un espace et un temps donné, ils se représentent par des hexagones avec un titre. Eux aussi peuvent s'imbriquer pour par exemple signifier l'appartenance d'une personne à une institution. De plus, les acteurs appartenant à plusieurs institutions pourront se placer à l'intersection de ces institutions à la manière des représentations logiques de Venn [THI 06], contrairement aux dimensions physiques qui elles ne peuvent pas faire partie de deux ensembles simultanément. Par exemple, dans la représentation cidessous, « Acteur 2 » appartient à la fois à « Institution 1 » et à «Institution 2 ». Un cercle est associé à l'acteur pour signifier l'intériorité de celui-ci, c'est dans ce cercle que prendront place les concepts liés à chaque acteur.

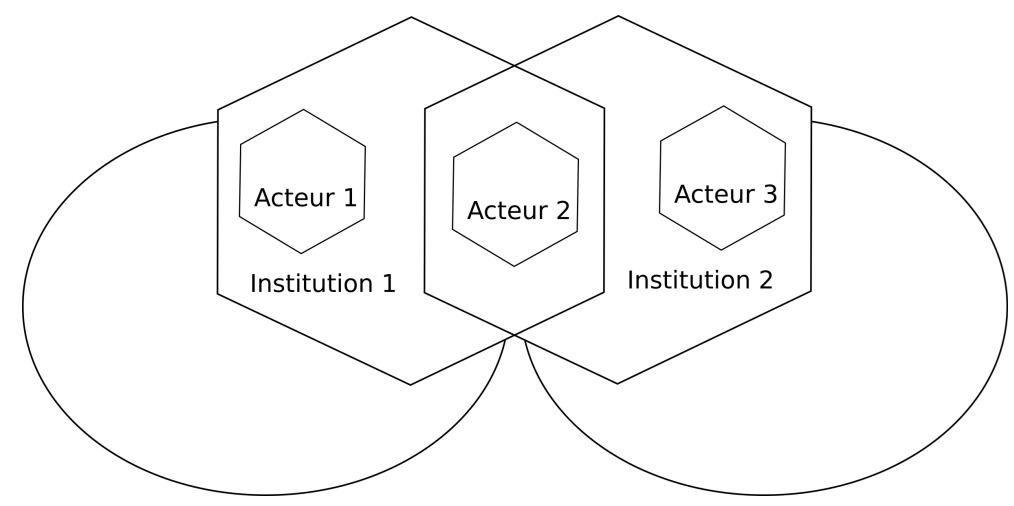

Figure 2. Modélisation des acteurs

\subsubsection{Les concepts}

Les concepts correspondent aux dimensions sémantiques de la modélisation, soit sous la forme d'un mot-clef voir sous la forme d'une phrase. Attention, un mot dans une dimension physique ne correspond pas à un concept dont le sens n'est pas réductible à une représentation et doit donc être nécessairement et perpétuellement renégocier avec soi-même et les autres. Il en découle que les modélisations ne sont finalement que les supports de ces négociations et n'ont pas pour vocation de représenter une vérité unique et absolue. 
Les concepts sont représentés par des cercles avec un titre. Tout comme les acteurs, ils peuvent se combiner pour se rassembler par groupe pour exprimer une dimension hiérarchique ou sous forme d'ensemble de Venn pour signifier des relations logiques ${ }^{3}$.

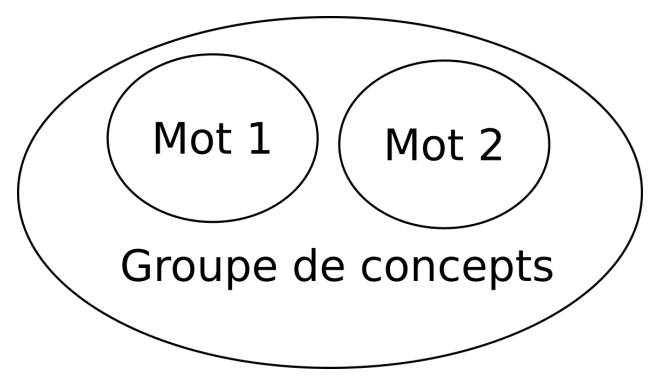

Figure 3. Modélisation des concepts

\subsubsection{Les rapports}

Les rapports représentent les relations des dimensions physiques et conceptuelles instanciées par un acteur dans un espace et un temps donné. Elles se figurent sous la forme de lignes simples pour représenter un état fini, ou sous la forme de lignes fléchées pour représenter un processus. Les rapports sont qualifiés par une source, une destination et un prédicat, ils peuvent être considérés comme des triplets RDF (sujet, prédicat, objet).

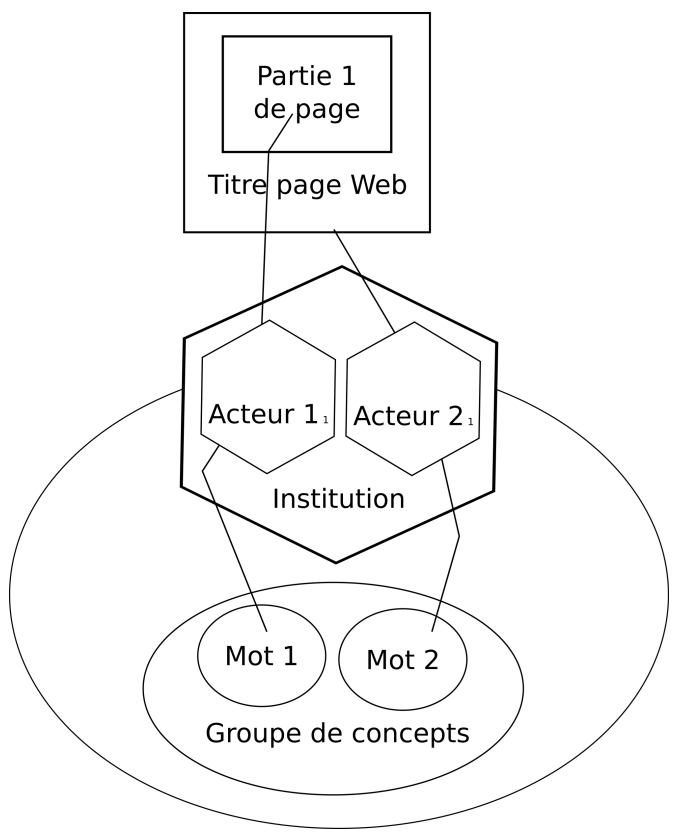

Figure 4. Modélisation de rapports finis

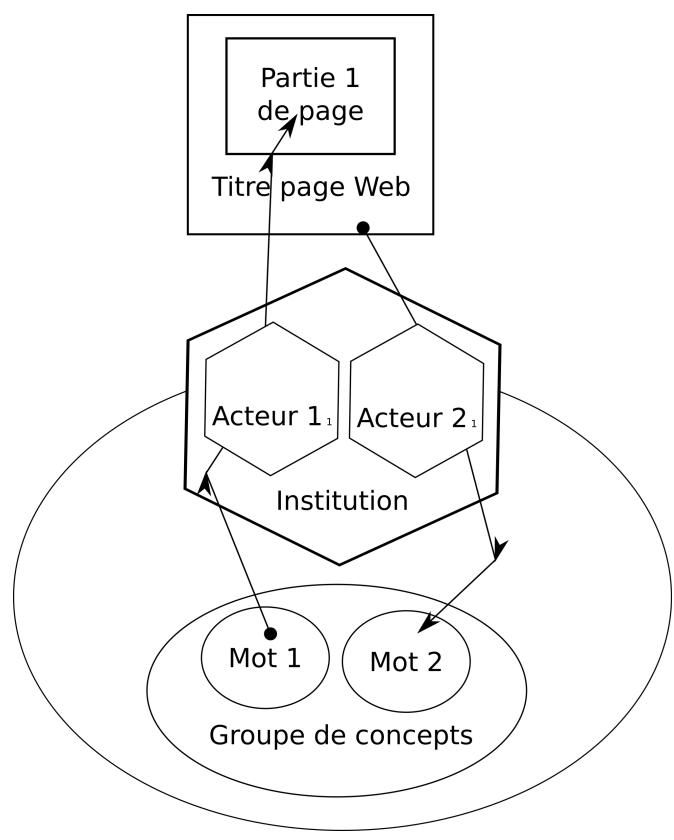

Figure 5. Modélisation de processus

C'est à travers les rapports que nous pouvons représenter le pouvoir d'agir d'une existence informationnelle dans un écosystème de connaissances. Ce pouvoir est bien évidemment mouvant et dépendra du contexte spatio-temporelle mais la modélisation permet d'en montrer les potentialités et de fournir aux analystes un support pour leurs questionnements et la formalisation de leurs hypothèses. De plus cette modélisation est très utile pour calculer la complexité de ce pouvoir.

\subsection{Calculer la complexité d'un écosystème}

A partir de ces principes de modélisation, il est possible de calculer la complexité d'un écosystème de connaissances suivant des règles génériques qui s'appliquent quel que soit le domaine de

\footnotetext{
${ }^{3}$ https://en.wikipedia.org/wiki/Venn_diagram
} 
connaissances concerné. Par la même, la comparaison des domaines devient possible en termes de complexité écosystémique et de complexité des points de vue sur l'écosystème analysé.

\subsubsection{Complexité existentielle}

Selon un principe de fractalité, les existences informationnelles complexes se composent d'existences informationnelles plus simples mais bâties sur le même modèle. La complexité d'une existence informationnelle est calculée à partir de la somme des dimensions physique, d'acteur, de concept et de rapport. Notons que cette complexité augmente lorsque les dimensions sont composées de sous partis qui décrivent le détail d'une dimension. Ainsi à la simple addition des parties, il faut ajouter un coefficient de fractalité qui multiplie le nombre de sous-partie par le niveau de détail.

Par exemple, si on modélise une bibliothèque comme une seule dimension physique, cette existence a une complexité de 1 , c'est à dire : 1 dimension physique * 1 niveau de fractalité $=1$. Si on ajoute dans la modélisation les livres qu'elle contient, on modélise un niveau supplémentaire de détail, le coefficient de fractalité passe donc à 2 . La complexité de ce nouveau modèle se calcule en additionnant la complexité initiale (1) et le nombre de livre que l'on multiplie par le coefficient de fractalité : 2 . Si la bibliothèque compte 100 livres, la complexité sera donc de $1+\left(100^{*} 2\right)=201$. Le même procédé s'applique si on ajoute au modèle le nombre de page de chaque livre, puis le nombre de mot et le nombre de caractère. Cet exemple ne prend en compte que les dimensions physiques de l'écosystème, il faut bien entendu reprendre le calcul pour les dimensions des acteurs, des concepts et des rapports. Ces derniers étant un cas particulier puisqu'il n'y a qu'un seul niveau pour les rapports car ceux-ci forment des réseaux dans lesquels il n'y a pas d'inclusion d'un nœud dans un autre. Le calcul de la complexité se fait sur la base du nombre d'élément distincts dans les 15 permutations possibles des 4 dimensions existentielles en 3 propriétés du rapport: source, destination et prédicat. A cette somme d'élément on ajoute la somme total de rapport de chaque permutation pour calculer une complexité qui augmentant avec le nombre de permutation et diminue avec le nombre de répétition d'un même élément dans une permutation (cf. ci-dessous pour un exemple de calcul).

\subsubsection{Complexité des points de vue}

Parallèlement à la complexité existentielle de l'écosystème de connaissance, on peut calculer la complexité du point de vue qu'un analyste porte sur cet écosystème. En effet, l'analyse d'un écosystème de connaissances ne peut être exhaustive celui-ci étant infiniment descriptible dans chaque dimension qui le compose. L'analyste devra donc faire des choix et ne prendre en compte qu'une partie de l'écosystème. Si nous reprenons l'exemple de la bibliothèque, le point de vue de l'analyste ne prend pas forcément en compte la totalité des livres mais uniquement un seul, voire une phrase dans un des livres ou même un mot. Dès lors, la complexité de son point de vue peut être calculé et comparé à d'autres points de vue sur le même écosystème. Un indice de complexité du point de vue peut même être calculé en faisant le rapport entre la complexité du point de vue sur la complexité de l'écosystème. Un point de vue idéal qui prendrait en compte la totalité d'un écosystème aurait donc un indice de 1.

Nous travaillons sur un prototype de plateforme pour l'exploration des écosystèmes de connaissances qui devrait faciliter la modélisation et automatiser le calcul des complexités existentielles et celles des points de vue. Cet outil n'est pas encore opérationnel mais voici une copie d'écran qui donne une idée de l'interface utilisateur. 


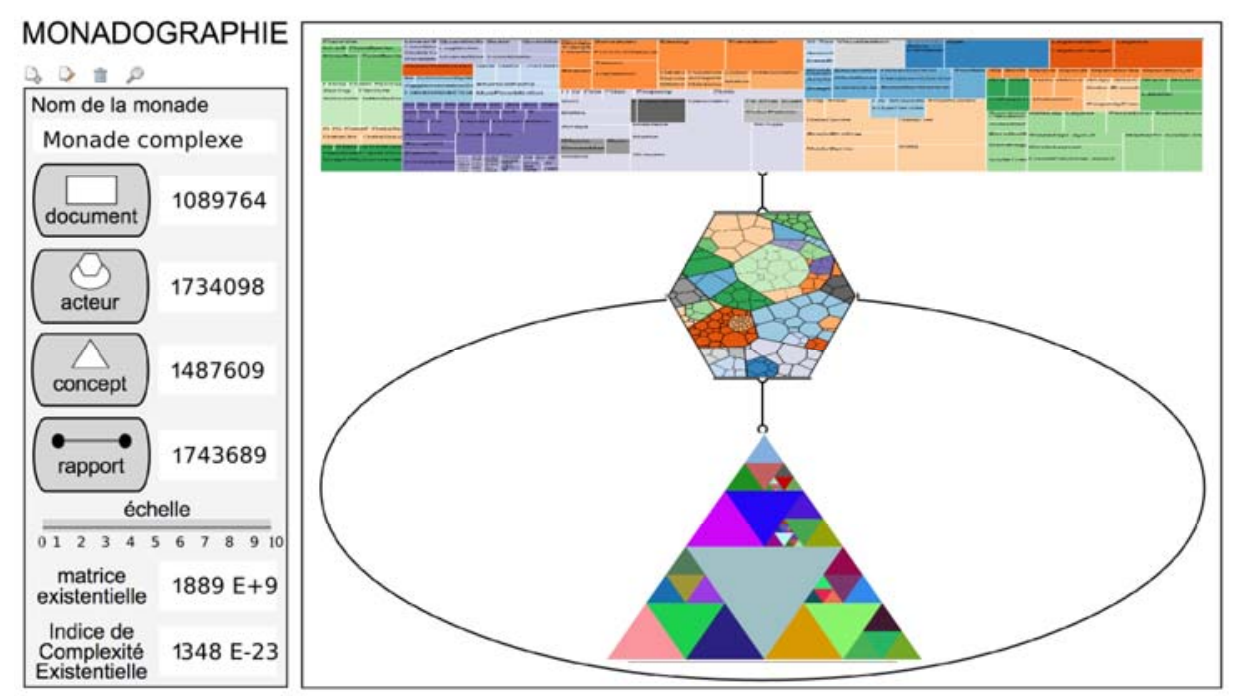

Figure 6. Outils d'exploration des écosystèmes de connaissances

Une fois finaliser cet outil pourra être utilisé pour par exemple calculer la complexité des points de vue d'une institution démocratique sur un problématique éthique comme la fait le parlement européen sur les règles concernant la robotique.

\section{Les règles de droit civil sur la robotique}

Dans le cadre d'une procédure d'initiative législative, le parlement européen a travaillé pendant un peu plus de deux ans (janvier 2015 à février 2017) sur des règles de droit civil concernant la robotique. Les rapports, les débats et les recommandations qui résultent de ces travaux, nous semblent un matériau particulièrement intéressant pour analyser la vision européenne concernant les enjeux de l'Internet des Objets. Plus globalement, ces travaux illustrent notre travail sur la modélisation des écosystèmes de connaissances et finalement nous donnent à évaluer la pertinence des procédures d'intelligence collective mises en place pour fabriquer un discours démocratique.

\subsection{Le corpus de données brutes}

Pour construire notre modèle, nous commencerons par décrire les méthodes que nous avons utilisées pour récolter le corpus que nous avons rassemblé. Il se compose dans un premier temps des documents Web que nous avons trouvés sur le site du parlement européen dédié à l'observation des procédures législatives.

Le point d'entrée documentaire de notre corpus et la page Web de l'Observatoire législatif dédié à la procédure d'initiative législative concernant la robotique ${ }^{4}$ (cf. figure 7). A partir de cette page nous pouvons réaliser une première modélisation qui reprend les informations importantes pour les représenter suivant nos règles de modélisation éthique et ainsi structurer la page en surlignant avec Diigo $^{5}$ ce qui relève des dimensions physiques en vert, d'acteurs en jaune, de concepts en rouge et de rapports en bleu (cf. figure 8).

\footnotetext{
${ }^{4}$ http://www.europarl.europa.eu/oeil/popups/ficheprocedure.do?lang=fr\&reference=2015/2103(INL)

${ }^{5}$ Diigo est un outil d'annotation, d'organisation et de partage de ressources Web : https://www.diigo.com/index 

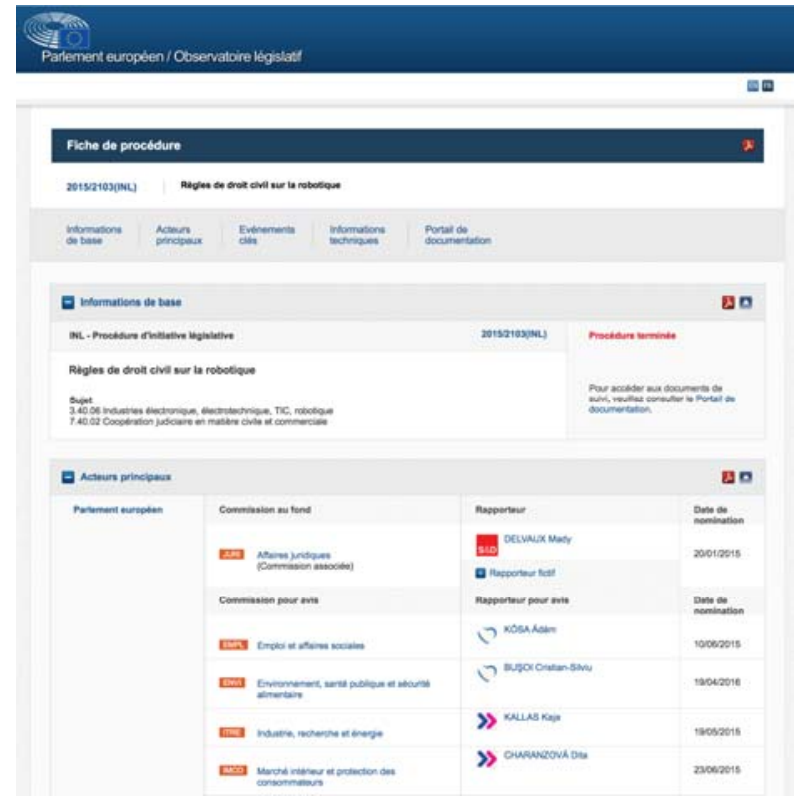

Figure 7. Page Web sans annotation

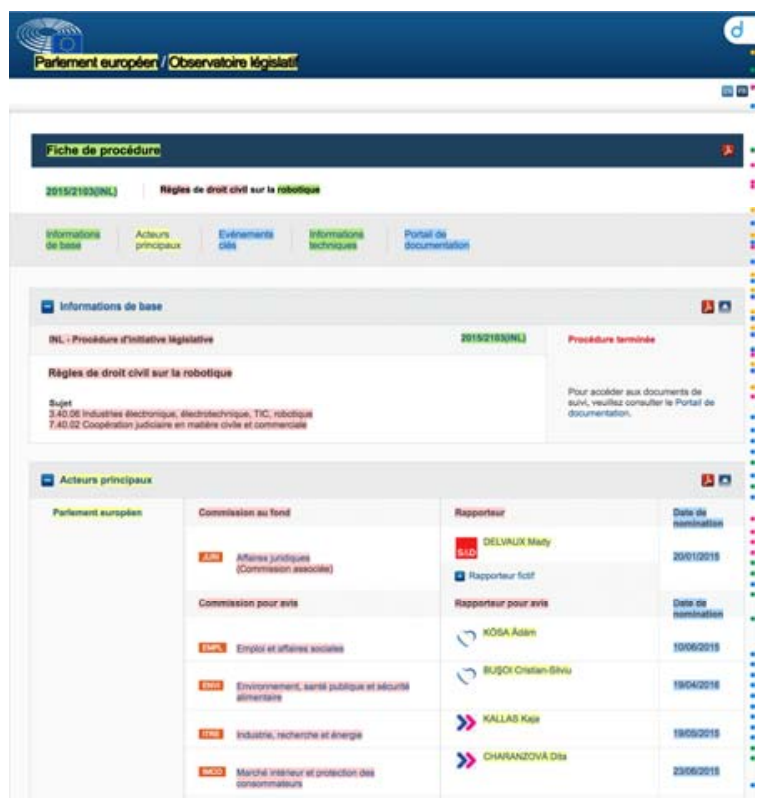

Figure 8. Page Web avec annotations Diigo ${ }^{6}$

Le recueil de ces annotations est consultable sur le portail Diigo qui les présente sous forme de liste avec la couleur choisie pour surligner (cf. Figure 9). Pour exploiter ces informations et les utiliser dans un processus de modélisation, on peut reprendre manuellement cette liste et construire le diagramme en faisant des copier / coller dans un outil de dessin vectoriel comme Inkscape ${ }^{7}$. Toutefois, dans le cas d'un écosystème très complexe, ce travail devient beaucoup trop fastidieux, voire même impossible.

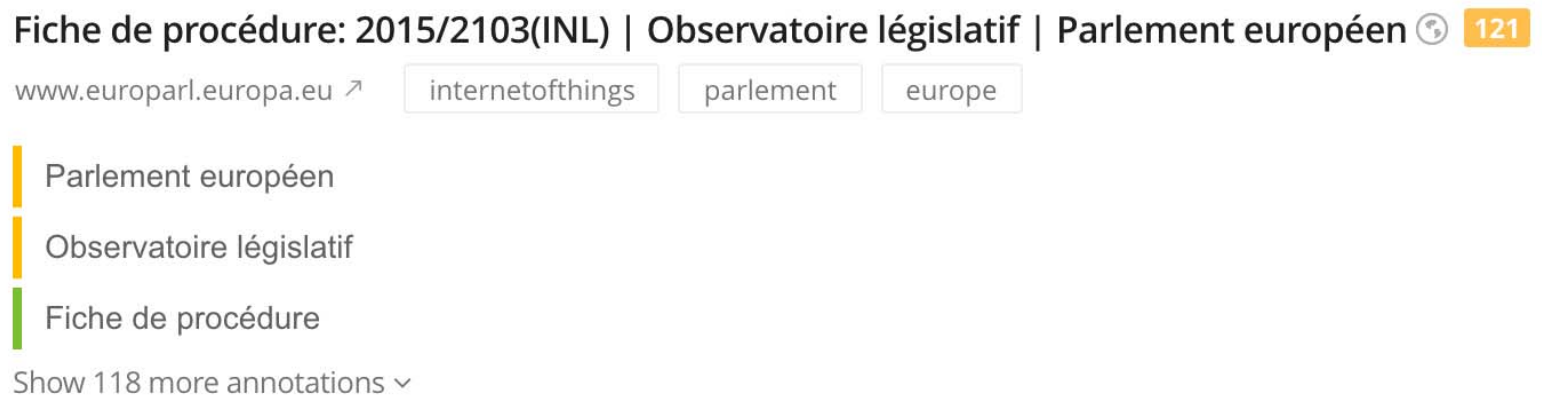

Figure 9. Liste des annotations sur le portail Diigo

Plusieurs scénarii sont envisageables pour automatiser, au moins en partie, la modélisation de cet écosystème mais l'algorithme dont nous avons besoin pour faire ce travail doit effectuer les actions suivantes :

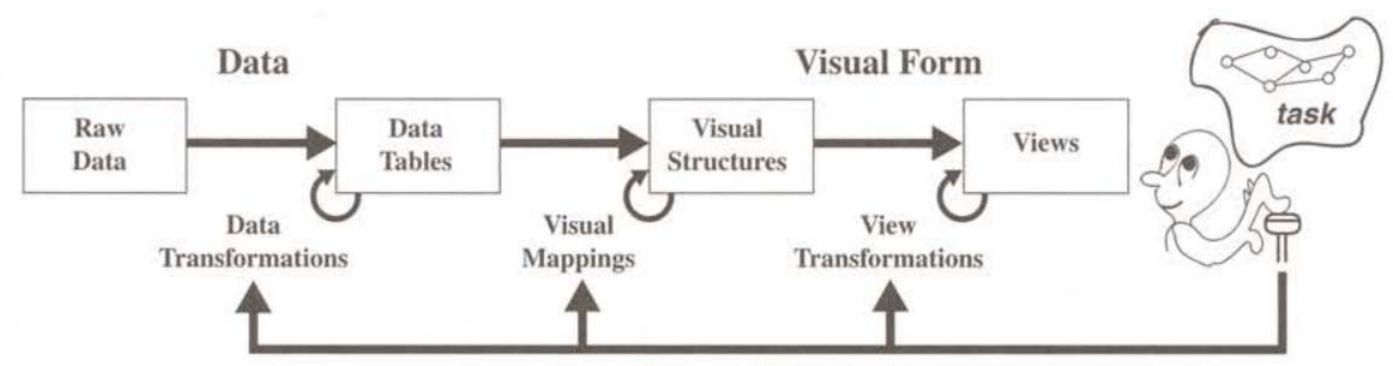

Human Interaction

Figure 10. Algorithme pour la modélisation des données [CARD 1999]

\footnotetext{
${ }^{6}$ lien vers la page annotée : https://diigo.com/09p0jf

${ }^{7}$ lien vers le site du logiciel : https://inkscape.org/fr/ 


\subsection{La transformation des données brutes}

Diigo est un outil très pratique pour annoter rapidement des documents Web mais pour récupérer les données brutes (Raw Data), l'utilisation de l'API de Diigo ${ }^{8}$ n'est pas totalement efficace car elle ne donne pas les informations concernant la couleur des annotations et donc la dimension existentielle auxquels nous les avons liés. Un algorithme d'extraction automatique des contenus (Web scraping) permettrait de récupérer ces informations toutefois dans le cas de l'observatoire législatif du parlement européen, il est plus efficace d'utiliser les propres services de cette institution (ParlTrack) pour récupérer toutes les informations d'une procédure législative sous une forme facilement exploitable par un algorithme 9 .

A partir de ce service, nous avons développé un algorithme pour récolter, catégoriser et stocker les informations concernant les débats au parlement européen pour les règles de droit civil sur la robotique. Cet algorithme ${ }^{10}$ prend comme source le fichier JSON $^{11}$ fourni par la plateforme ParlTrack pour enregistrer les informations dans une base de données dédiée à la modélisation des écosystèmes de connaissances. Nous avons construit l'algorithme à partir de l'organisation des informations proposée par ParlTrack. Nous avons développé 3 procédures principales permettant d'importer les activités, les amendements et les votes. Pour chacune de ces procédures nous avons créé les documents, les acteurs, les concepts et les rapports correspondants. Ces données structurées servent de base pour le calcul de la complexité de l'écosystème que nous résumons dans le tableau suivant :

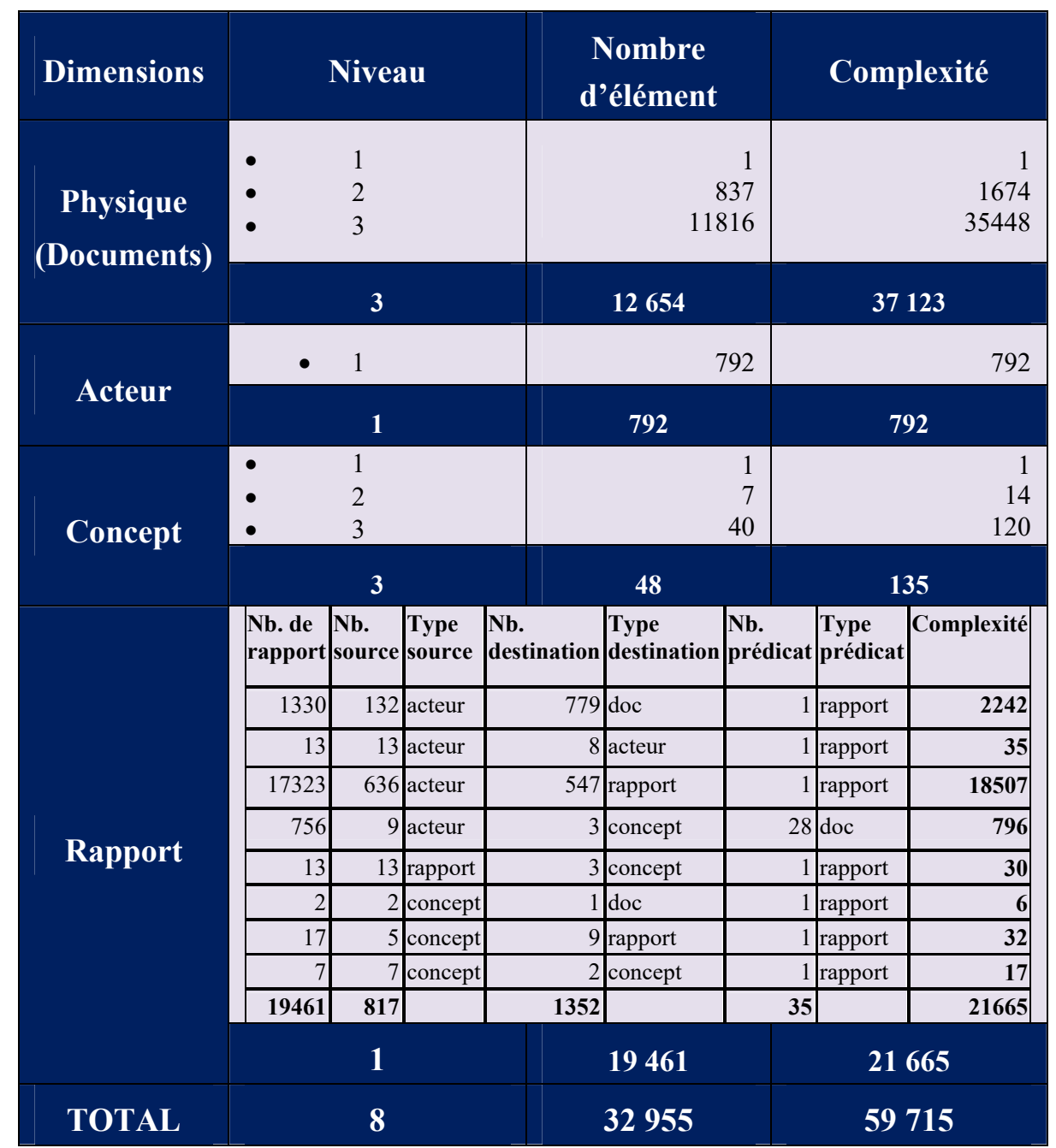

Tableau 1. Complexité de l'écosystème

\footnotetext{
${ }^{8}$ lien vers la documentation de l'API : https://www.diigo.com/api_dev

${ }^{9}$ lien vers l'API de suivi des procédures du parlement européen : http://parltrack.euwiki.org/

${ }^{10}$ lien vers le code PHP de l'algorithme : https://github.com/samszo/jardindesconnaissances/blob/master/library/Flux/Eu.php\#L81

${ }^{11}$ lien vers le fichier JSON : http://parltrack.euwiki.org/dossier/2015/2103(INL)?format=json

(c) 2017 ISTE OpenScience - Published by ISTE Ltd. London, UK - openscience.fr 
Les acteurs sont composés des rapporteurs, des votants et des groupes parlementaires. Il n'y a pas de hiérarchie car l'appartenance d'un parlementaire à un groupe est susceptible de changer, nous le traitons donc cette appartenance comme un rapport.

\section{Conclusion}

Cet article est une première étape dans le travail de recherche sur la modélisation éthique des écosystèmes de connaissances. Nous avons fixé les principes théoriques et les règles graphiques, récolté un premier corpus de données brutes, transformé ces données pour qu'elles correspondent avec le modèle à quatre dimensions que nous utilisons et finalement nous avons montré que cette modélisation permet un calcul du pouvoir d'agir d'un écosystème de connaissance.

Pour continuer cette recherche, nous allons exploiter la base de données que nous avons constitué pour l'enrichir par une analyse des documents qu'elle contient. En effet, pour l'instant la modélisation porte sur la structuration des données brutes mais pas sur leurs contenus. Il convient maintenant d'analyser dans les textes fournis par les différentes commissions, ce qui relève de chaque dimension existentielle. Par exemple, il serait très intéressant de connaître les éléments qui se ressemblent ou divergent dans les 4941 opinions qui composent les 806 amendements du débat.

Un autre point sur lequel nous devons avancer, concerne l'automatisation de la représentation du modèle. A l'aide de librairie JavaScript comme D3.js ${ }^{12}$, nous prévoyons de construire automatiquement un diagramme à partir d'une sélection de données pour fournir aux analystes un modèle de base qu'ils pourront faire évoluer suivant le point de vue qu'ils veulent défendre. Cette modélisation automatique permettrait aussi de montrer les évolutions historiques des rapports suivant les temporalité de leur instanciations. Avec ces premiers modèles, notre objectif et de pouvoir étalonner d'autres modélisations et parvenir ainsi à une modélisation de l'écosystème de connaissance de l'internet des objets où les différents points de vue seront représentés et comparables dynamiquement les uns avec les autres.

Plus globalement, on peut se demander si les procédures d'intelligence collective mises en place au parlement européen peuvent servir d'exemple pour construire des modélisations éthiques qui soient le résultat d'un travail collaboratif et surtout évolutif.

\section{Bibliographie}

[ARR 15] ARRUABARRENA, B., 2015. «L'écosystème numérique de la datavisualisation. » I2D - Information, données \& documents ME 52, 56-58.

[BaC 11] Bachimont, B., Gandon, F., Poupeau, G., Vatant, B., Troncy, R., Pouyllau, S., Martinez, R., BAtTisti, M., ZaCKLAD, M., 2011. «Enjeux et technologies : des données au sens ». Documentaliste-Sciences de l'Information 48, 24-41. DOI:10.3917/DOCSI.484.0024

[BOU 16] BouHä̈, N., HACHOUR, H., SALEH, I., 2016. Frontières numériques et artéfacts. Editions L'Harmattan, Paris.

[CAR 99] CARD, S.K., MACKINLAY, J.D., SHNEIDERMAN, B., 1999. Readings in Information Visualization, Morgan Kaufmann Publishers Inc., San Francisco, CA, USA, pp. 579-581.

[DEL 80] Deleuze, G., 1980. La voix de Gilles Deleuze - Spinoza - Des vitesses de la pensée [WWW Document]. URL http://www.univ-paris8.fr/deleuze/article.php3?id_article=91 (accessed 4.22.10).

[DES 05] DESCOLA, P., 2005. Par-delà nature et culture. NRF : Gallimard, Paris.

[THI 06] JÉRÔME THIÈVRE, 2006. Cartographies pour la Recherche et l'Exploration de données Documentaires (Informatique). MONTPELLIER II.

\footnotetext{
12 lien vers le site de la librairie : https://d3js.org/
} 
[YAN 17] JIANXIAO YANG, 2017. «Récepteur Multi-normes pour les Réseaux de Capteurs de l'IoT médical ». Internet des objets 1. doi:10.21494/ISTE.OP.2017.0136

[LAT 15] LATOuR, B., 2015. Face à Gä̈a. La Découverte, Paris.

[LAT 12] Latour, B., 2012. Enquêtes sur les modes d'existence: Une anthropologie des Modernes. Editions La Découverte, Paris.

[ALO 14] LyLia AlOUACHE, 2017. « Nouveau protocole robuste pour les communications dans 1'IoV ». Internet des objets 1. DOI:10.21494/ISTE.OP.2017.0139

[NOY 17] NOYER, J.-M., 2017. "L'Internet des Objets, l'Internet of "Everything" quelques remarques sur l'intensification du plissement numérique du monde ». Internet des objets 1. DOI:10.21494/ISTE.OP.2017.0139

[RAB 05] RABARDEL, P., 2005. «Instrument subjectif et développement du pouvoir d'agir », IN: Modèles Du Sujet Pour La Conception : Dialectiques, Activités, Développement. Octarès éditions, Toulouse.

[SAL 17] SALEH, I., 2017. «Les enjeux et les défis de l'Internet des Objets (IdO)». Internet des objets 1. DOI:10.21494/ISTE.OP.2017.0139

[SAL 14] SALEH, I., BOUHAI, N., HACHOUR, H., 2014. Les frontières du numérique. Editions L’Harmattan, Paris.

[SZO 15] SzONIECKY, S., 2015. «Interpréter la voix de Deleuze. Exemple de jardinage des connaissances », IN: Frontières D'archives Recherches, Mémoires, Savoirs. Editions des archives contemporaines, Paris, pp. 165-177.

[SZO 15] SZONIECKY, S., LOUÂPRE, M., 2015. «Outillages numériques pour les humanités : cartographier des réseaux d'influences », IN: ISKO - Magreb 2015. Presented at the Organisation de la Connaissance dans la perspective des Humanités Numériques : recherches et applications, Hammamet, Tunisie. 